\title{
Cytotoxic 20,22-Dihydrodigitoxigenin Glycosides and Other Constituents of Vallaris glabra Stems
}

Sudarat Kruakaew, Chonticha Seeka, Jantana Yahuafai, Pongpun Siripong, and Somyote

Sutthivaiyakit

\section{Supporting Information}

\section{Structural Identification of 7-10}

Compound 7 was isolated as an amorphous solid with molecular formulas of $\mathrm{C}_{42} \mathrm{H}_{68} \mathrm{O}_{18}$ based on its HRESIMS data. The ${ }^{1} \mathrm{H}$ and ${ }^{13} \mathrm{C}$ NMR spectra of 7 exhibited resonances rather similar to those of $\mathbf{3}$, except that $\mathbf{7}$ possesses an acofriopyranosyl unit instead of a vallarosyl group, together with recognizable resonances of two glucopyranosyl units in 7 (Table S1, Supporting Information), respectively. On the basis of previous study ${ }^{2}$ and $\mathrm{HMBC}$ spectra of $\mathbf{7}$ showing cross-peaks between $\mathrm{H}-1^{\prime} / \mathrm{C}-3, \mathrm{H}-1^{\prime \prime} / \mathrm{C}-4^{\prime}$ and $\mathrm{H}-1^{\prime \prime \prime} / \mathrm{C}-6^{\prime \prime}$, compound 7 was 20,22dihydrodigitoxigenin-3- $O$ - $\beta$-D-glucopyranos yl- $(1 \rightarrow 6)-\beta$-D-glucopyranosyl- $(1 \rightarrow 4)-\alpha$-Lacofriopyranoside.

Compound 8 was isolated as an amorphous solid exhibiting a molecular formula of $\mathrm{C}_{44} \mathrm{H}_{70} \mathrm{O}_{19}$. The ${ }^{1} \mathrm{H}$ and ${ }^{13} \mathrm{C}$ NMR spectra showed resonances similar to those of 7 (Table S1, Supporting Information), but with additional resonances of an acetyl group $\left(\delta_{\mathrm{H}} 2.10\right.$, s) and a less-shielded H-2' resonance at $\delta_{\mathrm{H}} 5.17(\mathrm{dd}, J=3.2$ and $1.8 \mathrm{~Hz})$. Based on the 2-D NMR data, 8 was thus proposed as 20,22 -dihydrodigitoxigenin-3- $O$ - $\beta$-D-glucop yranos yl- $(1 \rightarrow 6)$ - $\beta$-D-glucop yranos yl$(1 \rightarrow 4)-\alpha-\mathrm{L}-2$ '- $O$-acetylacofriopyranoside.

Compound 9 was obtained as an amorphous solid, its HRESIMS exhibited a $[\mathrm{M}+\mathrm{Na}]^{+}$ion at $m / z$ 897.4056 (calcd for $\mathrm{C}_{42} \mathrm{H}_{66} \mathrm{O}_{19} \mathrm{Na}, 897.4077$ ). The ${ }^{1} \mathrm{H}$ and ${ }^{13} \mathrm{C}$ NMR spectra showed rather complex sets of resonances, although the characteristic resonances of a gitoxigenin and vallarosyl groups could be recognized (Table S2, Supporting Information). Additional resonances, particularly of the two anomeric $\left[\delta_{\mathrm{H}} 4.39\left(\mathrm{~d}, J=7.8 \mathrm{~Hz}, \mathrm{H}-1^{\prime \prime}\right.\right.$, and $\left.\delta_{\mathrm{C}} 102.5, \mathrm{C}-1^{\prime \prime}\right)$ and $\delta_{\mathrm{H}} 4.39\left(\mathrm{~d}, J=7.8 \mathrm{~Hz}, \mathrm{H}-1^{\prime \prime \prime}\right.$, and $\left.\left.\delta_{\mathrm{C}} 105.0, \mathrm{C}-1^{\prime \prime \prime}\right)\right]$ and two oxymethylene groups $\left[\delta_{\mathrm{H}} 4.14\right.$ $(\mathrm{dd}, J=11.8$ and $1.9 \mathrm{~Hz}), \delta_{\mathrm{C}} 70.2, \mathrm{C}-6^{\prime \prime}$, and $\delta_{\mathrm{H}} 3.86(\mathrm{dd}, J=11.5$ and $1.8 \mathrm{~Hz})$, and $3.66(\mathrm{dd}, J=$ 11.5 and $5.3 \mathrm{~Hz}$ ) and $\left.\delta_{\mathrm{C}} 62.8, \mathrm{C}-6^{\prime \prime \prime}\right]$, indicated the presence of two glucosyl groups in 9 . The connectivities of $\mathrm{C}-3-O$ to $\mathrm{C}-1^{\prime}, \mathrm{C}-4^{\prime}-O$ to $\mathrm{C}-1^{\prime \prime}$ and $\mathrm{C}-6^{\prime \prime}-O$ to $\mathrm{C}-1^{\prime \prime \prime}$ were based on the HMC cross-peaks between $\mathrm{H}-1^{\prime} / \mathrm{C}-3$; H-1"/C-4', and $\mathrm{H}-1^{\prime \prime \prime} / \mathrm{C}-6^{\prime \prime}$, respectively. On the basis of previous report which provided L-vallarose, L-acofriose and $\mathrm{D}$-glucose after acid hydrolyses of oleandrigenin-3- $O-\alpha$-L-2'- $O$-acetylvallaropyranoside and oleandrigenin-3- $O-\beta$-D-

glucopyranosyl- $(1 \rightarrow 4)-\alpha$-L-2'- $O$-acetylacofriopyranoside, ${ }^{2} 9$ was thus proposed as gitoxigenin-3$O$ - $\beta$-D-glucopyranosyl- $(1 \rightarrow 6)-\beta$-D-glucopyranos yl-( $(1 \rightarrow 4)$ - $\alpha$-L-vallaropyranoside.

Compound $\mathbf{1 0}$ was isolated as an amorphous colorless solid with same molecular mass as of $\mathbf{9 .}$ The ${ }^{1} \mathrm{H}$ and ${ }^{13} \mathrm{C}$ NMR spectra exhibited resonances similar to those of $\mathbf{9}$ (Table S2, Supporting Information), although the resonances for a vallarosyl moiety were replaced by those of an 
acofriosyl group, showing an indicative resonance for $\mathrm{H}-5^{\prime}$ at somewhat higher-field than that of a vallarosyl group in $\mathbf{9}$. Based on its spectroscopic data and a previous study, $\mathbf{1 0}$ was therefore concluded to be gitoxigenin-3- $O$ - $\beta$-D-glucopyranosyl- $(1 \rightarrow 6)-\beta$-D-glucopyranosyl- $(1 \rightarrow 4)-\alpha$-Lacofriopyranoside.

\section{Isolation of Compounds 7-16}

\section{EXPERIMENTAL SECTION}

General Experimental Procedures. Melting points were measured using an Electrothermal melting point apparatus and are uncorrected. Optical rotations were recorded on a JASCO DIP 1020 polarimeter. The IR spectra were obtained on a Perkin-Elmer 1760x FT-IR spectrophotometer. The ${ }^{1} \mathrm{H}$ and ${ }^{13} \mathrm{C}$ NMR spectra were recorded with a Bruker AVANCE III HD $400 \mathrm{MHz}$ NMR spectrometer. Chemical shifts are referenced to the residual solvent signals $\left(\mathrm{MeOH}-d_{4}: \delta_{\mathrm{H}} 3.30\right.$ and $\left.\delta_{\mathrm{C}} 49.0 \mathrm{ppm}\right)$. HRESIMS were recorded on a Bruker Daltonic smicroTOF mass spectrometer.

Plant Material. The plant investigated, Vallaris glabra, was obtained as previously reported. A voucher specimen (SSVG-1/2012) is maintained at the Department of Chemistry, Ramkhamhaeng University. ${ }^{2}$

Extraction and Isolation. Dried V. glabra stems $(4.5 \mathrm{~kg})$ were ground and extracted successively with hexanes $(8 \mathrm{~L}), \mathrm{CH}_{2} \mathrm{Cl}_{2}(8 \mathrm{~L})$ and $\mathrm{MeOH}(8 \mathrm{~L})$, respectively, using a Soxhlet extractor. The resultant hexanes $(70.0 \mathrm{~g}), \mathrm{CH}_{2} \mathrm{Cl}_{2}(59.9 \mathrm{~g})$ and $\mathrm{MeOH}(95.2 \mathrm{~g})$ extracts were obtained after removal of solvent.

The $\mathrm{CH}_{2} \mathrm{Cl}_{2}$ extract (59.9 g) was fractionated by column chromatography (CC, silica gel, hexanes- $\mathrm{CH}_{2} \mathrm{Cl}_{2}$ 65:35 to $\mathrm{CH}_{2} \mathrm{Cl}_{2}-\mathrm{MeOH}$ 85:15) to obtain ten fractions. Fraction 3 (3.65 g) was fractionated by CC (Sephadex $\mathrm{LH}-20, \mathrm{MeOH}$ ) to give four subfractions (3.1-3.4). Subfraction $3.3(1.37 \mathrm{~g})$ was fractionated (CC, silica gel, hexanes-EtOAc 70-30) to give seven subfractions (3.3.1-3.3.7). Subfraction 3.3.1 provided ursolic acid $(30.8 \mathrm{mg})$ and subfraction 3.3 .2 provided 3,27-dihydroxyursolic acid (19.1 mg) after recrystallization from $\mathrm{CH}_{2} \mathrm{Cl}_{2} / \mathrm{MeOH}$. Subfraction 3.3.3 (134.0 mg) was purified using CC (silica gel, hexanes-EtOAc 70:30) to give three subfractions (3.3.3.1-3.3.3.3), and subfraction 3.3.3.2 (42.4 mg) was further purified by CC (silica gel, $\mathrm{CH}_{2} \mathrm{Cl}_{2}-\mathrm{MeOH}$ 99:1) to afford $\mathbf{1 5}$ ( $\left.8.3 \mathrm{mg}\right), \mathbf{1 1}(4.2 \mathrm{mg}$ ) and $\mathbf{1 3}(13.8 \mathrm{mg})$. Subfraction 3.3 .5 (190.3 mg) was subjected to CC (silica gel, hexanes-EtOAc 70:30) to give 16 $(22.0 \mathrm{mg})$. Fraction 4 (1.65 g), after fractionation (CC, Sephadex LH-20, MeOH), provided three subfractions (4.1-4.3). Subfraction $4.2(703.6 \mathrm{mg})$ was further fractionated (Sephadex LH-20, $\mathrm{MeOH}$, then $\mathrm{CC}$, silica gel, $\mathrm{CH}_{2} \mathrm{Cl}_{2}-\mathrm{MeOH}$ 98:2) to give additional amount of 11 (10.9 mg) and 13 (3.8 $\mathrm{mg})$, and also 14 (39.5 mg) and 12 (31.7 mg).

The $\mathrm{MeOH}$ extract $\left(95.2 \mathrm{~g}\right.$ ) was fractionated by $\mathrm{CC}$ (Dianion $\mathrm{HP}-20, \mathrm{MeOH}-\mathrm{H}_{2} \mathrm{O}, 0: 100$ to 100:0) to obtain five fractions. The water-soluble fractions 1-3, containing mostly sugars, were not investigated further. The less polar fraction $5(6.35 \mathrm{~g})$ was fractionated by reversed-phase CC (RP-18, MeOH- $\mathrm{H}_{2} \mathrm{O} 30: 70$ to 100:0) to obtain six subfractions (5.1-5.6). Subfraction 5.2 (820.3 $\mathrm{mg}$ ) was purified by $\mathrm{CC}$ (silica gel, $\mathrm{CH}_{2} \mathrm{Cl}_{2}-\mathrm{MeOH}, 88: 12$ to $85: 15$ ) and provided three subfractions (5.2.1- 5.2.3). Subfraction 5.2.2 (79.2 mg) after reversed-phase CC (RP-18, MeOH$\mathrm{H}_{2} \mathrm{O} 40: 60$ to 100:0) furnished oleandrigenin-3- $O$ - $\beta$-D-glucopyranosyl- $(1 \rightarrow 6)-\beta$-Dglucopyranosyl-( $(1 \rightarrow 4)-\alpha$-L-2'-O-acetylacofriopyranoside $(48.3 \mathrm{mg})$ and $8(4.2 \mathrm{mg})$. Subfraction $5.4(1.29 \mathrm{~g})$ was fractionated by $\mathrm{CC}\left(\mathrm{RP}-18, \mathrm{MeOH}-\mathrm{H}_{2} \mathrm{O} 10: 90\right.$ to 100:0) to give eight 
subfractions (5.4.1-5.4.8). Oleandrigenin-3- $O$ - $\beta$-D-glucopyranosyl-( $1 \rightarrow 6)$ - $\beta$-D-glucopyranos yl$(1 \rightarrow 4)$ - $\alpha$-L-vallaropyranoside ${ }^{2}(38.1 \mathrm{mg})$ was obtained from subfraction 5.4 .3 .4 . Subfraction 5.4.5 (147.1 mg) was $\mathrm{CC}$ (silica gel, $\mathrm{CH}_{2} \mathrm{Cl}_{2}-\mathrm{MeOH} 88: 12$ to 86:14, then RP-18, $\mathrm{MeOH}-\mathrm{H}_{2} \mathrm{O}$ $45: 55$ to $100: 0)$ to give oleandrigenin-3- $O$ - $\beta$-D-glucopyranosyl- $(1 \rightarrow 4)-\alpha$-L- $O$-acofriopyrano side $(11.2 \mathrm{mg})$ and oleandrigenin-3- $O$ - $\beta$-D-glucopyranosyl- $(1 \rightarrow 4)-\alpha$-L- $O$-vallaropyranoside $(2.3 \mathrm{mg})$. Subfraction 5.4.6 (243.5 mg) was purified by $\mathrm{CC}$ (silica gel, $\mathrm{CH}_{2} \mathrm{Cl}_{2}-\mathrm{MeOH} 88: 12$ to 86:14) to give five subfractions (5.4.6.1-5.4.6.5). Subfraction 5.4.6.5 (33.9 $\mathrm{mg})$ after further purification (CC, RP-18, MeOH-H ${ }_{2} \mathrm{O} 40: 60$ to 100:0) provided 7 (4.7 mg). Subfraction 5.4.8 (270.5 mg) was subjected to $\mathrm{CC}$ (silica gel, $\mathrm{CH}_{2} \mathrm{Cl}_{2}-\mathrm{MeOH} 85: 15$ to 80:20) to give four subfractions (5.4.8.15.4.8.4). Subfraction 5.4.8.1 (12.6 mg) afforded $3(2.9 \mathrm{mg})$ and $4(1.7 \mathrm{mg})$ after CC (RP-18, $\mathrm{MeOH}-\mathrm{H}_{2} \mathrm{O} 50: 50$ to 100:0). Subfraction 5.4.8.3 (29.0 mg) was further purified by CC (RP-18, $\mathrm{MeOH}-\mathrm{H}_{2} \mathrm{O} 55: 50$ to $\left.100: 0\right)$ to give additional quantity of $\mathbf{8}(6.3 \mathrm{mg})$. The polar fraction $4(2.25$ g) was fractionated using $\mathrm{CC}$ (silica gel, $\mathrm{CH}_{2} \mathrm{Cl}_{2}-\mathrm{MeOH} 88: 12$ to 84:16) to obtain eleven subfractions (4.1-4.11). Selection of compounds based on TLC identity led to 9 (5.3 mg) and $\mathbf{1 0}$ (4.0 mg) being obtained from subfraction $4.11(45.2 \mathrm{mg})$ after reversed-phase CC (RP-18, $\mathrm{MeOH}-\mathrm{H}_{2} \mathrm{O}$ 40:60 to 100:0).

20,22-Dihydrodigitoxigenin-3-O- $\beta$-D-glucopyranosyl-(1 $\rightarrow 6)$ - $\beta$-D-glucopyranosyl- $(1 \rightarrow 4)$ - $\alpha$ - $L$ acofriopyranoside (7): $[\alpha]^{25}-42.3$ (c 0.23, MeOH); FT-IR (ATR) $v_{\max } 3366,2922,2873,2855$, 1746, 1450, 1379, 1234, 1199, 1105, 1068, 1045, 1016, $986 \mathrm{~cm}^{-1} ;{ }^{1} \mathrm{H}$ NMR (MeOH- $d_{4}, 400$ $\mathrm{MHz})$ and ${ }^{13} \mathrm{C} \mathrm{NMR}\left(\mathrm{MeOH}-d_{4}, 100 \mathrm{MHz}\right)$ data see Table S1, Supporting Information; HRESIMS $m / z$ 883.4315 [M + Na] ${ }^{+}$(calcd for $\mathrm{C}_{42} \mathrm{H}_{68} \mathrm{NaO}_{18}, 883.4284$ ).

20,22-Dihydrodigitoxigenin-3-O- $\beta$-D-glucopyranosyl- $(1 \rightarrow 6)-\beta$-D-glucopyranosyl- $(1 \rightarrow 4)-\alpha-L$ 2'-O-acetylacofriopyranoside (8): $[\alpha]^{25}-40.7$ (c 0.34, MeOH); FT-IR (ATR) $v_{\max } 3369,2928$, 2884, 2865, 1732, 1723, 1447, 1376, 1236, 1214, 1122, 1094, 1067, 1038, 1019, $987 \mathrm{~cm}^{-1} ;{ }^{1} \mathrm{H}$ NMR $\left(\mathrm{MeOH}-d_{4}, 400 \mathrm{MHz}\right)$ and ${ }^{13} \mathrm{C} \mathrm{NMR}\left(\mathrm{MeOH}-d_{4}, 100 \mathrm{MHz}\right)$ data see Table S1, Supporting Information; HRESIMS $m / z, 925.4407[\mathrm{M}+\mathrm{Na}]^{+}$(calcd for $\mathrm{C}_{44} \mathrm{H}_{70} \mathrm{NaO}_{19}, 925.4389$ ).

Gitoxigenin-3-O- $\beta$-D-glucopyranosyl- $(1 \rightarrow 6)-\beta$-D-glucopyranosyl- $(1 \rightarrow 4)-\alpha$ - $L$ vallaropyranoside (9): $[\alpha]^{25}{ }_{\mathrm{D}}-35.9$ ( c 0.26, MeOH); FT-IR (ATR) $v_{\max } 3333,2922,2882,2858$, 1732, 1627, 1603,1453, 1349, 1269, 1165, 1068, 1027, $1014 \mathrm{~cm}^{-1} ;{ }^{1} \mathrm{H}$ NMR (MeOH- $d_{4}, 400$ $\mathrm{MHz})$ and ${ }^{13} \mathrm{C}$ NMR (MeOH- $\left.d_{4}, 100 \mathrm{MHz}\right)$ data see Table S2, Supporting Information; HRESIMS $m / z$ 897.4056 [M + Na] $]^{+}$(calcd for $\mathrm{C}_{42} \mathrm{H}_{66} \mathrm{NaO}_{19}, 897.4077$ ).

Gitoxigenin-3-O- $\beta$-D-glucopyranosyl- $(1 \rightarrow 6)-\beta$-D-glucopyranosyl- $(1 \rightarrow 4)-\alpha-L$ acofriopyranoside (10): $[\alpha]_{\mathrm{D}}^{25}-26.4$ (c 0.22, MeOH); FT-IR (ATR) $v_{\max } 3359,2922,2854$, 1730, 1627, 1449, 1377, 1288, 1243, 1164, 1103, 1068, 1023, $987 \mathrm{~cm}^{-1}$; ${ }^{1} \mathrm{H}$ NMR (MeOH- $d_{4}$, $400 \mathrm{MHz})$ and ${ }^{13} \mathrm{C} \mathrm{NMR}\left(\mathrm{MeOH}-d_{4}, 100 \mathrm{MHz}\right)$ data see Table S2, Supporting Information; HRESIMS $m / z$ 897.4062 [M + Na] ${ }^{+}$(calcd for $\mathrm{C}_{42} \mathrm{H}_{66} \mathrm{NaO}_{19}, 897.4077$ ). 
Table S1. ${ }^{1} \mathrm{H}(400 \mathrm{MHz})$ and ${ }^{13} \mathrm{C}(100 \mathrm{MHz})$ NMR Spectroscopic Data of 7 and 8 (in MeOH- $d_{4}$ )

\begin{tabular}{|c|c|c|c|c|}
\hline \multirow[b]{2}{*}{ position } & \multicolumn{2}{|l|}{7} & \multicolumn{2}{|l|}{8} \\
\hline & $\delta_{\mathrm{H}}(\mathrm{J}$ in $\mathrm{Hz})$ & $\delta_{\mathrm{C}}$, type & $\delta_{\mathrm{H}}(\mathrm{J}$ in $\mathrm{Hz})$ & $\delta_{\mathrm{C}}$, type \\
\hline 1 & $1.74,1.38$ & $30.9, \mathrm{CH}_{2}$ & $1.83,1.55$ & $31.0, \mathrm{CH}_{2}$ \\
\hline 2 & $1.78,1.15$ & 27.9, $\mathrm{CH}_{2}$ & $1.90,1.25$ & $27.4, \mathrm{CH}_{2}$ \\
\hline 3 & $3.83 \mathrm{brs}\left(\mathrm{W}_{1 / 2}=7.5 \mathrm{~Hz}\right)$ & 74.0, $\mathrm{CH}$ & $3.93\left(\mathrm{~W}_{1 / 2}=8.0 \mathrm{~Hz}\right)$ & 74.7, $\mathrm{CH}$ \\
\hline 4 & $1.42,1.30$ & 31.6, $\mathrm{CH}_{2}$ & 1.39 & $31.6, \mathrm{CH}_{2}$ \\
\hline 5 & 1.51 & $38.2, \mathrm{CH}$ & 1.65 & $38.2, \mathrm{CH}$ \\
\hline 6 & 1.48 & $27.4, \mathrm{CH}_{2}$ & 1.58 & $27.8, \mathrm{CH}_{2}$ \\
\hline 7 & 1.66 & $22.4, \mathrm{CH}_{2}$ & 1.77 & $22.4, \mathrm{CH}_{2}$ \\
\hline 8 & 1.56 & $42.3, \mathrm{CH}$ & 1.64 & $42.4, \mathrm{CH}$ \\
\hline 9 & 1.60 & $36.8, \mathrm{CH}$ & 1.64 & $36.4, \mathrm{CH}$ \\
\hline 10 & - & $36.5, \mathrm{C}$ & - & $36.4, \mathrm{C}$ \\
\hline 11 & $1.28,1.15$ & $22.1, \mathrm{CH}_{2}$ & $1.25,1.35$ & 22.1, $\mathrm{CH}_{2}$ \\
\hline 12 & $1.33,1.25$ & $42.0, \mathrm{CH}_{2}$ & $1.33,1.42$ & $42.0, \mathrm{CH}_{2}$ \\
\hline 13 & - & $48.5, \mathrm{C}$ & - & $48.3, \mathrm{C}$ \\
\hline 14 & - & $86.8, \mathrm{C}$ & - & $86.8, \mathrm{C}$ \\
\hline 15 & $1.88,1.45$ & $32.4, \mathrm{CH}_{2}$ & $1.98,1.56$ & $32.4, \mathrm{CH}_{2}$ \\
\hline 16 & $1.83,1.43$ & $25.3, \mathrm{CH}_{2}$ & $1.93,1.51$ & $26.2, \mathrm{CH}_{2}$ \\
\hline 17 & 1.62 & $55.3, \mathrm{CH}$ & 1.73 & $55.3, \mathrm{CH}$ \\
\hline 18 & $0.88 \mathrm{~s}$ & $16.9, \mathrm{CH}_{3}$ & $0.94 \mathrm{~s}$ & $16.9, \mathrm{CH}_{3}$ \\
\hline 19 & $0.87 \mathrm{~s}$ & $24.4, \mathrm{CH}_{3}$ & $0.96 \mathrm{~s}$ & $24.4, \mathrm{CH}_{3}$ \\
\hline 20 & 2.77 quint-like (8.7) & $40.4, \mathrm{CH}$ & 2.86 quint-like (8.8) & $40.3, \mathrm{CH}$ \\
\hline 21 & $4.33 \mathrm{t}(8.7), 4.00 \mathrm{t}(9.1)^{a}$ & 73.9, $\mathrm{CH}_{2}$ & $4.41 \mathrm{t}(8.8), 4.08 \mathrm{t}(9.1)$ & 73.9, $\mathrm{CH}_{2}$ \\
\hline 22 & $\begin{array}{l}2.57 \mathrm{dd}(17.6,8.5) \\
2.15 \mathrm{dd}(17.6,10.0)\end{array}$ & $36.5, \mathrm{CH}_{2}$ & $\begin{array}{l}2.65 \mathrm{dd}(17.6,9.9), \\
2.23 \mathrm{dd}(17.6,8.5)\end{array}$ & $36.5, \mathrm{CH}_{2}$ \\
\hline 23 & - & $180.8, \mathrm{C}$ & - & 180.7, C \\
\hline $1^{\prime}$ & $4.71 \mathrm{~d}(2.0)$ & $99.8, \mathrm{CH}$ & $4.79 \mathrm{~d}(1.8)$ & $97.3, \mathrm{CH}$ \\
\hline $2^{\prime}$ & $3.87 \mathrm{dd}(2.0,3.1)$ & $68.5, \mathrm{CH}$ & $5.17 \mathrm{dd}(3.2,1.8)$ & $70.2, \mathrm{CH}$ \\
\hline $3^{\prime}$ & $3.50 \mathrm{dd}(3.1,8.9)$ & $82.6, \mathrm{CH}$ & $3.72 \mathrm{dd}(9.4,3.2)^{d}$ & $81.0, \mathrm{CH}$ \\
\hline $4^{\prime}$ & $3.64 \mathrm{t}(8.9)^{b}$ & $79.0, \mathrm{CH}$ & $3.62 \mathrm{t}(9.4)$ & $79.4, \mathrm{CH}$ \\
\hline $5^{\prime}$ & $3.62 \mathrm{dq}(8.9,5.8)^{b}$ & $68.7, \mathrm{CH}$ & $3.75 \mathrm{dq}(6.3,9.4)^{d}$ & $68.8, \mathrm{CH}$ \\
\hline $6^{\prime}$ & $1.18 \mathrm{~d}(5.4)$ & $18.3, \mathrm{CH}_{3}$ & $1.29 \mathrm{~d}(6.3)$ & $18.3, \mathrm{CH}_{3}$ \\
\hline $\mathrm{OCH}_{3}$ & $3.34 \mathrm{~s}$ & $56.3, \mathrm{CH}_{3}$ & $3.39 \mathrm{~s}$ & $57.7, \mathrm{CH}_{3}$ \\
\hline $1^{\prime \prime}$ & $4.51 \mathrm{~d}(7.8)$ & $104.9, \mathrm{CH}$ & $4.58 \mathrm{~d}(7.8)$ & $104.9, \mathrm{CH}$ \\
\hline $2^{\prime \prime}$ & $3.11 \mathrm{t}(8.4)$ & $75.2, \mathrm{CH}$ & $3.14 \mathrm{t}(8.6)$ & 75.6, $\mathrm{CH}$ \\
\hline 3" & 3.25 & $77.9, \mathrm{CH}$ & $3.36 \mathrm{t}(8.9)^{e}$ & $77.7, \mathrm{CH}$ \\
\hline $4^{\prime \prime}$ & 3.24 & $71.6, \mathrm{CH}$ & $3.34 \mathrm{t}(9.2)^{e}$ & $71.8, \mathrm{CH}$ \\
\hline $5^{\prime \prime}$ & $3.29^{c}$ & $77.0, \mathrm{CH}$ & 3.42 ddd $(5.7,3.5,1.9)$ & $76.9, \mathrm{CH}$ \\
\hline
\end{tabular}




$\begin{array}{lllll}6^{\prime \prime} & 4.04 \mathrm{dd}(11.8,1.8)^{a}, 3.67^{b} & 70.5, \mathrm{CH}_{2} & 4.13 \mathrm{dd}(11.7,1.9), & 70.5, \mathrm{CH}_{2} \\ & & & 3.78 \mathrm{dd}(11.7,5.7)^{d} & \\ 1^{\prime \prime \prime} & 4.28 \mathrm{~d}(7.8) & 105.0, \mathrm{CH} & 4.37 \mathrm{~d}(7.8) & 105.0, \mathrm{CH} \\ 2^{\prime \prime \prime} & 3.08 \mathrm{t}(8.1) & 75.7, \mathrm{CH} & 3.21 \mathrm{dd}(8.9,7.8) & 75.2, \mathrm{CH} \\ 3^{\prime \prime \prime} & 3.25^{c} & 77.8, \mathrm{CH} & 3.36 \mathrm{t}(8.9)^{e} & 78.0, \mathrm{CH} \\ 4^{\prime \prime \prime} & 3.24 \mathrm{t}(6.9) & 71.8, \mathrm{CH} & 3.27 \mathrm{t}^{f} & 71.6, \mathrm{CH} \\ 5^{\prime \prime \prime} & 3.17 & 77.9, \mathrm{CH} & 3.26^{f} & 78.0, \mathrm{CH} \\ 6^{\prime \prime \prime} & 3.77 \mathrm{~d}(11.1), & 62.7, \mathrm{CH}_{2} & 3.86 \mathrm{dd}(12.1,1.7), & 62.8, \mathrm{CH}_{2} \\ & 3.58 \mathrm{dd}(5.0,11.7) & & 3.66 \mathrm{dd}(11.9,5.1) & \\ \mathrm{OCOCH}_{3} 2^{\prime} & & & 2.10 \mathrm{~s} & 20.9, \mathrm{CH}_{3} \\ & & & & 172.2, \mathrm{C}^{\prime}\end{array}$

${ }^{a-f}$ Overlapped signals. 
Table S2. ${ }^{1} \mathrm{H}(400 \mathrm{MHz})$ and ${ }^{13} \mathrm{C}(100 \mathrm{MHz})$ NMR Spectroscopic Data of 9 and 10 (in MeOH$\left.d_{4}\right)$

\begin{tabular}{|c|c|c|c|c|}
\hline \multirow[b]{2}{*}{ position } & \multicolumn{2}{|c|}{9} & \multicolumn{2}{|c|}{10} \\
\hline & $\delta_{\mathrm{H}}(J$ in $\mathrm{Hz})$ & $\delta_{\mathrm{C}}$, type & $\delta_{\mathrm{H}}(J$ in $\mathrm{Hz})$ & $\delta_{\mathrm{C}}$, type \\
\hline 1 & $1.87,1.47$ & $31.5, \mathrm{CH}_{2}$ & $1.87,1.47$ & $31.6, \mathrm{CH}_{2}$ \\
\hline 2 & 1.60 & $27.9, \mathrm{CH}_{2}$ & 1.60 & $27.4, \mathrm{CH}_{2}$ \\
\hline 3 & $3.92\left(\mathrm{~W}_{1 / 2}=8.0 \mathrm{~Hz}\right)^{a}$ & $74.8, \mathrm{CH}$ & $3.94\left(\mathrm{~W}_{1 / 2}=9.0 \mathrm{~Hz}\right)$ & $74.1, \mathrm{CH}$ \\
\hline 4 & $1.84,1.52$ & $31.5, \mathrm{CH}_{2}$ & $1.52,1.40$ & $31.0, \mathrm{CH}_{2}$ \\
\hline 5 & 1.77 & $38.1, \mathrm{CH}$ & 1.64 & $38.2, \mathrm{CH}$ \\
\hline 6 & $1.98,1.26$ & 27.6, $\mathrm{CH}_{2}$ & 1.90 & $27.8, \mathrm{CH}_{2}$ \\
\hline 7 & 1.43 & $22.5, \mathrm{CH}_{2}$ & $1.43,1.24$ & $22.1, \mathrm{CH}_{2}$ \\
\hline 8 & 1.58 & $42.9, \mathrm{CH}$ & 1.60 & $42.9, \mathrm{CH}$ \\
\hline 9 & 1.66 & $36.8, \mathrm{CH}$ & 1.66 & $36.8, \mathrm{CH}$ \\
\hline 10 & - & $36.3, \mathrm{C}$ & - & $36.3, \mathrm{C}$ \\
\hline 11 & $1.80,1.24$ & $22.1, \mathrm{CH}_{2}$ & 1.83 & $22.4, \mathrm{CH}_{2}$ \\
\hline 12 & $1.56,1.40$ & $41.0, \mathrm{CH}_{2}$ & $1.55,1.39$ & $41.0, \mathrm{CH}_{2}$ \\
\hline 13 & - & $51.3, \mathrm{C}$ & - & $51.3, \mathrm{C}$ \\
\hline 14 & - & $85.7, \mathrm{C}$ & - & $85.6, \mathrm{C}$ \\
\hline 15 & $\begin{array}{l}2.61 \mathrm{dd}(8.4,14.8) \\
1.70 \mathrm{dd}(2.3,14.8)\end{array}$ & $43.8, \mathrm{CH}_{2}$ & $\begin{array}{l}2.62 \mathrm{dd}(8.5,15.0) \\
1.70 \mathrm{dd}(2.2,15.0)\end{array}$ & $43.8, \mathrm{CH}_{2}$ \\
\hline 16 & $4.64 \mathrm{dd}(8.0,2.0)^{b}$ & 73.2, $\mathrm{CH}$ & $4.64 \mathrm{dt}(7.9,2.3)^{e}$ & 73.1, $\mathrm{CH}$ \\
\hline 17 & $3.12 \mathrm{~d}(7.8)$ & $57.9, \mathrm{CH}$ & $3.12 \mathrm{~d}(7.9)$ & $59.7, \mathrm{CH}$ \\
\hline 18 & $0.91 \mathrm{~s}$ & 17.1, $\mathrm{CH}_{3}$ & $0.91 \mathrm{~s}$ & $17.1, \mathrm{CH}_{3}$ \\
\hline 19 & $0.94 \mathrm{~s}$ & $24.4, \mathrm{CH}_{3}$ & $0.95 \mathrm{~s}$ & $24.4, \mathrm{CH}_{3}$ \\
\hline 20 & - & 173.6, C & - & 173.6, C \\
\hline 21 & $\begin{array}{l}5.16 \mathrm{dd}(1.6,16.8) \\
5.09 \mathrm{dd}(1.6,16.9)\end{array}$ & 77.9, $\mathrm{CH}_{2}$ & $\begin{array}{l}5.16 \mathrm{dd}(18.4,1.7) \\
5.09 \mathrm{dd}(18.5,1.6)\end{array}$ & 77.9, $\mathrm{CH}_{2}$ \\
\hline 22 & $5.93 \mathrm{t}(1.6)$ & $120.6, \mathrm{CH}_{2}$ & $5.93 \mathrm{t}(1.6)$ & 120.6, $\mathrm{CH}_{2}$ \\
\hline 23 & - & 177.3, C & - & 177.3, C \\
\hline $1^{\prime}$ & $4.66 \mathrm{~d}(2.9)^{b}$ & $100.3, \mathrm{CH}$ & $4.80 \mathrm{~d}(1.7)$ & $99.8, \mathrm{CH}$ \\
\hline $2^{\prime}$ & $3.93 \mathrm{dd}(5.4,3.4)^{a}$ & $69.3, \mathrm{CH}$ & $3.97 \mathrm{dd}(3.1,1.9)$ & $68.6, \mathrm{CH}$ \\
\hline $3^{\prime}$ & $3.63 \mathrm{dd}(5.2,3.3)$ & 79.0, $\mathrm{CH}$ & $3.59 \mathrm{dd}(8.9,3.2)$ & 82.7, $\mathrm{CH}$ \\
\hline $4^{\prime}$ & $3.95 \mathrm{dd}(7.6,3.2)$ & $76.8, \mathrm{CH}$ & $3.72^{f}$ & 79.1, CH \\
\hline $5^{\prime}$ & 4.20 quintet (7.1) & $67.2, \mathrm{CH}$ & $3.71^{f}$ & $68.8, \mathrm{CH}$ \\
\hline $6^{\prime}$ & $1.24 \mathrm{~d}(6.6)$ & $17.9, \mathrm{CH}_{3}$ & $1.27 \mathrm{~d}(5.3)$ & $18.3, \mathrm{CH}_{3}$ \\
\hline $\mathrm{OCH}_{3}$ & $3.48 \mathrm{~s}$ & $59.7, \mathrm{CH}_{3}$ & $3.43 \mathrm{~s}$ & $59.9, \mathrm{CH}_{3}$ \\
\hline $1^{\prime \prime}$ & $4.39 \mathrm{~d}(7.8)^{c}$ & $102.5, \mathrm{CH}$ & $4.60 \mathrm{~d}(7.8)^{e}$ & 104.9, CH \\
\hline $2^{\prime \prime}$ & $3.21 \mathrm{t}(7.9)$ & $75.2, \mathrm{CH}$ & $3.16 \mathrm{t}(8.6)$ & $75.8, \mathrm{CH}$ \\
\hline
\end{tabular}




\begin{tabular}{|c|c|c|c|c|}
\hline $3^{\prime \prime}$ & $3.36 \mathrm{t}(8.8)^{d}$ & $77.7, \mathrm{CH}$ & $3.34 \mathrm{t}(8.6)$ & $77.9, \mathrm{CH}$ \\
\hline $4^{\prime \prime}$ & $3.31^{d}$ & 71.7, $\mathrm{CH}$ & $3.33 \mathrm{t}(9.5)$ & 71.9, $\mathrm{CH}$ \\
\hline $5^{\prime \prime}$ & $3.45 \mathrm{ddd}(8.4,6.3,2.0)$ & $77.3, \mathrm{CH}$ & $3.40 \mathrm{ddd}(9.8,4.9,2.6)$ & $77.0, \mathrm{CH}$ \\
\hline 6" & $4.14 \mathrm{dd}(11.8,1.9)$ & $70.2, \mathrm{CH}_{2}$ & $\begin{array}{l}4.13 \mathrm{dd}(11.6,1.9) \\
3.75 \mathrm{dd}(11.7,6.0)^{f}\end{array}$ & $70.5, \mathrm{CH}_{2}$ \\
\hline $1^{\prime \prime \prime}$ & $4.39 \mathrm{~d}(7.8)^{c}$ & 105.0, $\mathrm{CH}$ & $4.37 \mathrm{~d}(7.8)$ & $105.0, \mathrm{CH}$ \\
\hline $2^{\prime \prime \prime}$ & $3.19 \mathrm{t}(7.9)$ & $75.3, \mathrm{CH}$ & $3.19 \mathrm{t}(7.8)$ & $75.2, \mathrm{CH}$ \\
\hline $3^{\prime \prime \prime}$ & $3.36^{d}$ & $77.8, \mathrm{CH}$ & $3.34 \mathrm{t}(7.7)$ & 77.9, $\mathrm{CH}$ \\
\hline $4^{\prime \prime \prime}$ & 3.28 & 71.7, CH & $3.28^{g}$ & 71.6, CH \\
\hline $5^{\prime \prime \prime}$ & 3.26 & $78.0, \mathrm{CH}$ & $3.26^{g}$ & $78.0, \mathrm{CH}$ \\
\hline $6^{\prime \prime \prime}$ & $\begin{array}{l}3.86 \mathrm{dd}(11.5,1.8) \\
3.66 \mathrm{dd}(11.5,5.3)\end{array}$ & $62.8, \mathrm{CH}_{2}$ & $\begin{array}{l}3.85 \mathrm{dd}(11.9,1.8), \\
3.66 \mathrm{dd}(11.9,5.3)\end{array}$ & $62.8, \mathrm{CH}_{2}$ \\
\hline
\end{tabular}

Table S3. Cytotoxic Activity of Compounds 11-16

\begin{tabular}{|c|c|c|c|c|}
\hline compound & HT-29 & A 549 & $\mathrm{HeLa}$ & Vero \\
\hline 11 & $>10$ & $2.3 \pm 0.1$ & $6.1 \pm 0.8$ & $>10$ \\
\hline 12 & $>10$ & $2.2 \pm 0.3$ & $8.0 \pm 1.6$ & $>10$ \\
\hline 13 & $0.6 \pm 0.1$ & $>10$ & $>10$ & $>10$ \\
\hline 14 & $>10$ & $6.5 \pm 0.8$ & $7.2 \pm 0.5$ & $>10$ \\
\hline 15 & $>10$ & $>10$ & $>10$ & $>10$ \\
\hline 16 & $>10$ & $6.8 \pm 0.1$ & $9.8 \pm 0.01$ & $>10$ \\
\hline doxorubicin $^{b}$ & $0.44 \pm 0.15$ & $1.0 \pm 0.1$ & $0.12 \pm 0.05$ & $3.5 \pm 0.7$ \\
\hline \multicolumn{5}{|c|}{$\begin{array}{l}{ }^{a} \text { Values indicated are } \mathrm{IC}_{50} \text { values in } \mu \mathrm{M} \text {, data are means } \pm \mathrm{SD} \text { of three independent } \\
\text { experiments, each performed in six replicates. }{ }^{b} \text { Positive control }\end{array}$} \\
\hline
\end{tabular}

